\title{
Are There Enough Data to Safely Withdraw Anticoagulation After Atrial Fibrillation Catheter Ablation?
}

\author{
Nikhil Ahluwalia, Richard Schilling \\ Department of Cardiac Electrophysiology, St Bartholomew's Hospital, London, UK
}

A trial fibrillation catheter ablation (AFCA) can significantly reduce the symptoms and total burden of the arrhythmia. Whether it can sufficiently reduce the associated thromboembolic risk to warrant discontinuation of anticoagulation in successful cases, is an important issue with impact on the increasing number of patients undergoing the procedure. Observational data suggests that successful AFCA may reduce the risk, but with the increasingly prevalent diagnosis of asymptomatic atrial fibrillation (AF), it needs careful consideration, especially as the value of anticoagulation in this setting is incompletely understood. In the absence of randomised trial data, expert consensus recommends continued risk stratification according to baseline risk factors, independent of procedural outcome. Despite this, a number of electrophysiologists routinely discontinue anticoagulation in moderate- to high-risk patients. Detailed counselling, prolonged intensive monitoring periods and outcome-based decision-making tools have supported these operators to keep their published stroke rates low. Anticoagulation conveys an inherent risk of major bleeding which is also seen with anti-platelet therapies, making this an illogical 'step-down' therapy. The risks of both, a continuation or discontinuation strategy, support the randomised study of anticoagulation after AFCA which is ongoing. However, the observational nature of the currently available data is insufficient to support change in practice. Whilst further results are awaited, our duty is to support patient decision-making explaining that, ultimately, best practice is currently unknown. The objective of this review is to critically analyse the existing data regarding discontinuation in this setting and also to consider the additional factors that may influence clinical decision-making on this contended issue.

\section{Keywords}

Atrial fibrillation, catheter ablation, anticoagulation, bleeding, asymptomatic AF, thromboembolic risk, stroke

Disclosure: Nikhil Ahluwalia and Richard Schilling have nothing to disclose in relation to this article.

Review Process: Double-blind peer review.

Compliance with Ethics: This study involves a review of the literature and did not involve any studies with human or animal subjects performed by any of the authors.

Authorship: The named authors meet the International Committee of Medical Journal Editors (ICMJE) criteria for authorship of this manuscript, take responsibility for the integrity of the work as a whole, and have given final approval for the version to be published.

Received: 23 May 2019

Accepted: 4 September 2019

Citation: European Journal of Arrhythmia

\& Electrophysiology. 2019;5(2):82-6

Corresponding Author: Nikhil Ahluwalia. Department of Cardiac Electrophysiology, St Bartholomew's Hospital, West Smithfield, London, EC1A 7BE.

E: nikhil.ahluwalia@nhs.net

Support: No funding was received in

the publication of this article.
The global disease burden of atrial fibrillation (AF) is considerable, and a significant proportion is attributable to the associated thromboembolic risk. The pro-thrombotic environment resulting from AF accrues a five-fold increase in stroke risk, with the arrhythmia implicated in 30\% of all strokes.1,2 Evaluating thromboembolic risk should be a primary concern for any healthcare practitioner responsible for a patient with $\mathrm{AF}^{3.4}$ Fortunately, this vulnerability can largely be mitigated with the timely prescription of oral anticoagulation therapy (OAT). Large-scale randomised controlled trial data has shown that OAT can significantly reduce thromboembolic events in at-risk patients with non-valvular AF. ${ }^{5}$ Although warfarin is the traditionally prescribed OAT, direct oral anticoagulants (DOACS) have become an increasing part of contemporary practice with similar, if not superior efficacy. ${ }^{6-8}$ Their desirable anti-thrombotic effect inadvertently predisposes takers to an increased risk of bleeding. Major bleeding events such as intracranial haemorrhage and gastro-intestinal bleeds are associated with greater morbidity in the context of OAT, although potentially less so with DOACs than warfarin. ${ }^{~}$ Nevertheless, these risks need to be considered when prescribing these drugs.

Risk stratification scores have been developed for the AF population to aid the counselling process for patients in whom OATs are being considered. The CHADS2 or CHA2DS2-VASC scores, and the HASBLED scores are probably the most commonly used for thromboembolic and bleeding risk assessment. ${ }^{10-2}$ These tools have been validated in AF populations managed using non-invasive rate control and/or rhythm control strategies.

The pro-thrombotic pathophysiology in AF is complex. Observational data suggests an association between AF burden and stroke risk, and it is intuitive to think that the effective restoration of sinus rhythm would reduce this risk. ${ }^{13,14}$ Medication-based rhythm control strategies have not demonstrated significant impact on thromboembolic risk..$^{15}$ The question therefore arises whether the restoration and maintenance of sinus rhythm with ablation will impact stroke prevention. The purpose of this review is to critically analyse the observation data regarding discontinuation of OAT in this setting. We will also consider the implications of asymptomatic AF (AAF) and cardiac devices on risk stratification.

Anticoagulation practice after atrial fibrillation catheter ablation AF catheter ablation (AFCA) has evolved as an effective therapy for treating symptomatic AF in selected patients. ${ }^{16}$ It is more effective than anti-arrhythmic medications at achieving sustained 
sinus rhythm. ${ }^{17}$ It leads to a marked reduction in total AF burden in experienced hands. ${ }^{18}$ Regarding anti-coagulation, best practice in the short-term following AFCA is largely agreed upon, with consensus supporting OAT for at least 2-3 months following the procedure. ${ }^{19}$ Although there is little evidence justifying this approach, it is generally felt that resolution and endothelialisation of ablation lesions, reduction in post-operative inflammation and recovery of atrial function may be important factors exposing the patient to an increased risk of thrombosis in the early post-ablation period that OAT may protect from. Reverse remodelling occurs although this is not always associated with return of active atrial function. Thus, the implications on longer-term thromboembolic risk are less clear. Whether procedural success can shift the thromboembolic-bleeding risk balance sufficiently to advocate long-term discontinuation of OAT, is the topic of ongoing study and debate.

Current guidelines recommend indefinite continuation of OAT based on thromboembolic risk assessment scores, regardless of the perceived procedural outcome. ${ }^{4,19}$ Discontinuation of OAT after AFCA is a commonly held patient expectation and occasionally is the primary incentive for undergoing the procedure. ${ }^{20}$ In a 2012 survey, 16\% (11/68) of European electrophysiologists reported they would discontinue OAT in $\leq 12$ months after a successful AFCA in a patient with CHA2DS2-VASC score of $2 .^{21}$ This divergent practice is mirrored by a similar proportion of surveyed Canadian electrophysiologists. ${ }^{22}$ This may reflect an individual interpretation of the inconsistent data differently informing different operators' practice.

Increasing numbers of centres are performing rising numbers of AFCA procedures. The issue of how to best manage this expanding cohort of successfully ablated patients is significant and was highlighted as an important unanswered question in the most recent International expert consensus statement on AFCA. ${ }^{19}$ An overview of the relevant literature is important to inform practice. In the absence of consensus, this review will also consider the factors that can support personalised decision-making.

\section{The impact of atrial fibrillation catheter ablation on thromboembolic risk}

Jarman et al. undertook a retrospective observational analysis $(n=4,991)$ in an attempt to characterise the longitudinal impact of AFCA on stroke risk compared to an extensively propensity-matched cohort managed medically. ${ }^{23}$ Repeated measures methodology demonstrated a similar baseline annual incidence of stroke $(0.30 \%$ versus $0.28 \%, p=0.829)$ but with significant divergence over the 5 years following the indexed AFCA, cardioversion or hospital visit $(0.64 \%$ versus $1.84 \%, p<0.001)$. These findings would suggest AFCA can convey an attractive risk reduction that persists over many years. However, the influence of selection bias through unmatched variables on this disparity should be considered. Outcome detection was also dependent on a health-record key-word search which may under-represent the true incidence.

Two large, prospective case series have independently reported a significantly lower stroke risk in populations that underwent AFCA versus age- and gender-matched AF cohorts treated medically. ${ }^{24,25}$ Over 3-years follow-up, both series reported the incidence of stroke after AFCA became comparable to matched populations with no known AF. Hunter et al. further found that 'freedom from $\mathrm{AF}^{\prime}$ ' was an independent predictor of stroke-free survival after multi-variate analysis (hazard ratio $[\mathrm{HR}] \quad 0.33$; 95\% confidence interval $[\mathrm{Cl}]$ 0.17-0.67) potentially delineating a dependent relationship with procedural outcome.25 However, longer-term observational data suggests this ablation outcome-dependent association may not endure over time with a comparable 10-year stroke risk between those who remain in sinus rhythm and those who do not (HR 0.79; 95\% $\mathrm{Cl} 0.48-1.29 ; \mathrm{p}=0.34) .{ }^{26}$

Although the three aforementioned studies are observational with inherent limitations as a result of their design, the cohorts undergoing AFCA reflect clinical practice. The absolute annual incidence of stroke reported in these cohorts is very low and comparable to cohorts in lifelong sinus rhythm. However, the standard practice in these studies was to manage thromboembolic risk according to their pre-procedural risk score regardless of procedural success. A contemporary retrospective analysis of Canadian administrative data suggests the risk reduction in the post-AFCA period may be attributable to better OAT compliance; an unmatched variable in these studies. ${ }^{27}$ The authors reported a significant reduction in event rate (stroke/transient ischaemic attack/retinal infarct) after AFCA compared to a propensity-matched, medically managed AF cohort (HR 0.43; 95\% Cl 0.37-0.50). However, it became comparable after adjustment for OAT use (HR 0.88; 95\% Cl 0.63-1.21). This study too, however, was observational and may be subject to its own selection bias. There was a difference in baseline stroke risk between the unadjusted control and AFCA (CHA2DS2-VASC scores $3.2 \pm 1.6$ versus $1.1 \pm 1.2$ groups potentially due to selection bias that improved after adjustment for OAT use $(2.3 \pm 1.4$ versus $2.2 \pm 1.3)$.

In attempt to better control for such features, a large, randomised controlled trial compared AFCA to medical management, with the incidence of stroke over 4 years reported as a secondary end-point. ${ }^{28}$ The CABANA investigators reported no significant difference in the prevalence of disabling stroke at follow-up (HR 0.42; 95\% Cl 0.11-1.62; $\mathrm{p}=0.19$ ). The study was, however, underpowered to detect a difference and this finding should be read with this in consideration. Despite enrolling 2,204 patients over 4 years, this multicentre trial remained underpowered for this endpoint due to the very low event rate in both the intervention and medication arms (0.3\% versus $0.6 \%)$. OAT prescription was at the physician's discretion and almost all participants were taking one form at time of follow-up (95\% versus $96 \%$ ). This may have diluted any difference in effect by contributing to the low event rates in both groups.

\section{Discontinuation of oral anticoagulation therapy in the post-atrial fibrillation catheter ablation period}

OAT discontinuation after AFCA is routinely practised in a number of centres who have published their experiences. Saad et al. published a series of 327 patients who were switched from OAT to anti-platelet therapy in the absence of any suspicion of recurrence 3 months after AFCA (and resumed if AF was detected) ${ }^{29} \mathrm{~A}$ total of $225(68.8 \%)$ of these patients had a CHADS2 score of 2-3. No strokes were noted over a mean follow-up period of $46 \pm 17$ months whilst off OAT, suggesting strategic feasibility.

The largest reported case series was published by Themistoclakis et al., from five centres that discontinued OAT after AFCA as standard. ${ }^{30}$ They used a standardised 'opt-in' criterion of high-risk features (atrial tachyarrhythmia recurrence, absent a-wave on pulsed Doppler trans-mitral flow analysis or severe pulmonary vein stenosis) to identify patients to continue on OAT. In total, 663 (19.7\%) patients were continued 'on-OAT', predominantly due to arrhythmia recurrence. Otherwise patients were switched to aspirin at a median of 4 months $(5 \pm 3)$ post-procedure $(n=3,355)$. A total of $723(27 \%)$ had one CHADS2 risk factor and 347 (13\%) had two or more. Ten of these subjects had CHADS2 scores of $5-6$. Over a mean follow up of $28 \pm 3$ months, $2(0.07 \%)$ 
thromboembolic events occurred in the 'off-OAT' group and 3 (0.45\%) in the 'on-OAT' group. The incidence was not significantly different $(p=0.06)$, although any inter-cohort comparison is of limited value given the inherent differences between the two groups. However, the study did demonstrate two key points; discontinuation of OAT may be feasible after AFCA as standard protocol without any clear increase in thromboembolic events, and that a simple criteria-based tool may identify patients in whom to continue OAT.

The incidence of stroke in both studies remained low after AFCA despite OAT discontinuation with short-term outcomes comparable to observational case series wherein it was continued. These findings favour discontinuation in patients with medium to high risk and suggests AFCA could be a predominant driver for risk reduction.

A study of OAT discontinuation exclusively in high-risk patients has demonstrated similar short-term outcomes. ${ }^{31}$ One-hundred-and-eight patients undergoing AFCA with a prior stroke and an average CHA2DS2-VASC score of $4.1 \pm 1.4$ were enrolled. The authors chose to implement a prolonged post-procedural surveillance period with repeated rhythm checks to more confidently determine procedural outcome. After a mean period of 7.3 months, 55 patients were subsequently diagnosed as free of recurrence and, after counselling, discontinued OAT. Although the sample size of this single-centre study was small, no strokes were reported over the $2.2 \pm 1.3$-year follow-up period, supporting the observational body of data. Meta-analysis of nine observational studies $(n=3,436)$ of OAT continuation versus discontinuation after AFCA found no significant difference in thromboembolic events in patients at moderate or greater risk at baseline. ${ }^{32}$

\section{Bleeding risk of oral anticoagulation therapy continuation}

The bleeding risk associated with OAT must be cautiously considered in every patient with AF. The same holds for the re-assessment of short-term and long-term risk in the post-ablation period. The incidence, morbidity and mortality of major bleeding events are significantly increased on OAT, with intracranial haemorrhage and gastro-intestinal bleeding the most significant. ${ }^{33}$ Large randomised controlled trial data has suggested an annual bleeding risk of 2.1-3.6\% with warfarin and $3.1-3.4 \%$ with DOACs. ${ }^{6-8,34} \mathrm{~A}$ meta-analysis of these DOAC-versus-warfarin trials suggests a lower bleeding risk with DOAC use, although agent reversal and interactions represent different challenges that need consideration. ${ }^{35}$

Secondary prevention with OAT after a stroke is not advocated in patients with sinus rhythm. AF itself is not associated with greater bleeding risk. The prevalence and burden of thromboembolic events due to AF means a high bleeding risk alone should not lead to withholding of OAT. ${ }^{4}$ However, this recommendation cannot be held if the bleeding risk is unbalanced. Bleeding risk stratification tools in the setting of OAT prescription for AF have been established to aid the physician to identify and address associated risk factors. ${ }^{10}$ Although periprocedural bleeding is a recognised complication of AFCA, there has not been any data to suggest the procedure increases longer-term risk of major bleeding. The CABANA trial showed comparable rates of serious bleeding between the ablation and medically managed cohorts who largely continued OAT over the 4-year follow-up period (HR 0.98; 95\% $\mathrm{Cl} 0.62-1.56$; $\mathrm{p}=0.93$ ). ${ }^{28}$

Many of the above experiences reported bleeding event rates as a secondary outcome and found either a significantly greater incidence associated with OAT continuation or no difference. ${ }^{29-31}$ The greatest disparity in major bleeding was reported by Themistoclakis et al., with 13 events in the on-OAT cohort compared to two (2.0\% versus $0.3 \%$, $\mathrm{p}<0.001$ ). ${ }^{30} \mathrm{~A}$ meta-analysis of nine such studies reporting on OAT discontinuation after AFCA found a significantly higher incidence of major bleeding events in those who were continued on OAT. (OR 6.5; 95\% $\mathrm{Cl}$ 1.93-21.86; $\mathrm{p}=0.002$ ). ${ }^{36}$ However the lack of randomised data is again the limitation. Risk factors for bleeding and thromboembolism overlap (age, hypertension, previous stroke) and thus by continuing OAT in high thromboembolic risk patients may compound an inherently higher risk of bleeding.

The published series opted to switch OAT for life-long aspirin after the discontinuation of OAT. ${ }^{29,30}$ However, aspirin is not an anti-coagulant and there is no strong evidence to suggest it is effective preventative therapy against thromboembolic events in patients with AF. A meta-analysis of the relevant randomised trials comparing aspirin to OAT in AF patients demonstrated an inferiority of the anti-platelet in reducing thromboembolic events despite a comparable bleeding risk to novel OAT across all risk groups. ${ }^{37}$ European guidelines issue a class III recommendation for aspirin in this cohort due to the unjustified bleeding risk - even in low-risk patients. ${ }^{4}$ For patients in sinus rhythm, anti-platelet therapy does have a role in the secondary prevention of vaso-occlusive events associated with acute coronary syndromes and cerebrovascular accidents. As primary prevention in this cohort, its value is limited even amongst the elderly or high-risk. Thus, the routine prescription in place of OAT is not logical - if there is suspicion of arrhythmia recurrence, or if the clinician chooses to manage the patient according their pre-AFCA risk, OAT should be the therapy of choice. If OAT is to be discontinued, evidence would only support the substitution with aspirin if there is an alternative indication. Whether such a personalised approach would have led to a lower bleeding risk following OAT discontinuation thus further skewing the thromboembolic-bleeding risk is unknown but would be a valuable insight from future studies in this field.

\section{Implications of arrhythmia recurrence on thromboembolic risk}

AF can recur after AFCA and the potential unmitigated thromboembolic risk of AAF recurrence is a concern when considering OAT discontinuation. AAF is not unique to the post-AFCA population and has been documented both in patients known, and not known, to have AF. ${ }^{18,38}$ Identification in the latter often happens after a thromboembolic event or during routine interrogation of an otherwise-indicated cardiac implantable electronic device (CIED). ${ }^{39}$ The findings have reiterated that $A F$ is a continuous variable rather than existing in two dichotomous states (present or absent). Although CIEDs are able to capture even brief episodes of AAF ( $<2$ minutes), these do not appear to convey significant stroke risk. The minimum duration required to do so varies across the literature with 6-minute and 24-hour episodes of AAF reported as the lower thresholds. ${ }^{13,40}$ Further uncertainty exists regarding the onward management of AAF. Whether OAT can significantly reduce the thromboembolic risk associated with this phenomenon has not yet been established, questioning the merit of intensive surveillance post-AFCA.

The absolute AF burden is substantially reduced after AFCA as well as the duration of any paroxysmal episodes. The ratio of AAF:symptomatic $A F$, however, increases; dissociating symptoms from the arrhythmia. ${ }^{18}$ No predictor of AAF vulnerability has been identified although the duration of AF pre-ablation and number of AFCA procedures are independent predictors. ${ }^{39,41}$ 
Conventional symptom-directed follow-up may underestimate the true prevalence of arrhythmia recurrence. However, these undetected episodes of AAF tend to be much shorter than symptomatic AF and usually of duration below that associated with thromboembolic risk. ${ }^{41,42}$ AAF is uncommon in the absence of concurrent symptomatic AF and whether their detection would significantly alter risk and management needs to be determined. However, recurrence after AFCA may be a progressive phenomenon with AAF being the initial phase, evolving into longer, symptomatic episodes. ${ }^{18}$ And although early detection of AAF may not alter acute thromboembolic risk, it may forewarn of impending significant arrhythmias of sufficient duration to warrant re-commencement of OAT. ${ }^{43}$ A current paucity of data limits any consensus on the management of $A A F$, which is becoming a significant clinical phenomenon thanks to implantable devices and their remote follow up, detection and memory capabilities.

CIEDS present a novel paradigm for targeted OAT prescription during paroxysms of AF only facilitated by the advent of direct OAT agents with quick onset. This would avoid the unmitigated increase in bleeding risk during periods of sinus rhythm. Two trials, REACT.COM $(n=59)$ and TACTIC-AF $(n=48)$ have recently demonstrated the feasibility of a device-guided strategy in their small studied cohorts which included patients post-AFCA. ${ }^{44,45}$ Whereas REACT.COM reported a $94 \%$ reduction in time on OAT using a 1-hour threshold for episode significance, TACTIC-AF reported a 75\% reduction using a 6-hour threshold. Although this presents an attractive, personalised approach to risk stratification in this sub-group, outstanding concerns such as the AF episode duration threshold to trigger OAT commencement, the duration of therapy required per episode, and importantly whether the approach can significantly impact thromboembolic outcomes, limits wider application. The single randomised controlled trial assessing superiority of device-guided OAT versus standard of care for patients with dual chamber and biventricular defibrillators, was terminated early due to futility ${ }^{46}$ It demonstrated no significant difference in thromboembolic or major bleeding outcomes. However, an historic diagnosis of AF was not an inclusion criterion and participants predominantly had concomitant heart failure by virtue of their device indication. A better understanding of the association, including the temporal relationship, between AF episodes and thrombogenesis is required before considered application in carefully selected patients.

\section{Conclusion}

Observational series have demonstrated a reduced incidence of thromboembolic events in cohorts undergoing AFCA compared to matched counterparts. Centres routinely practising in OAT discontinuation after AFCA have reported the feasibility of adjusted follow-up protocols and no associated increased risk in their cohorts. The association between AAF and stroke is being more precisely defined, and this uncertainty underlies a primary concern of routine discontinuation. Smart-devices offer a potential non-invasive, remote monitoring tool that could enable more reactive decision-making; however, this would require high-sensitivity devices and effective patient autonomy. Importantly, large randomised controlled trials to determine the impact of OAT on AAF-associated stroke risk are already underway and their outcomes are eagerly awaited to help guide practice. ${ }^{47,48}$

The international expert consensus papers recognise the potential incongruity of OAT in the presence of sustained sinus rhythm and the need for further evidence. ${ }^{4,19}$ However, in the absence of any prospective randomised controlled trial data supporting discontinuation, this recommendation is unlikely to be revised. A prospective multi-centre, randomised controlled trial is underway, recruiting over 1,500 patients in hope to provide clarity on this issue (NCT02168829). ${ }^{49}$ Following AFCA for symptomatic $A F$, enrolled patients will be followed up with two 24-hour Holter over 12 months, and in the absence of any symptoms or episodes of atrial tachyarrhythmia $\geq 30$ seconds will be considered to be in sustained sinus rhythm. Enrolled patients with CHA2DS2-VASC $\geq 1$ will then be randomised to continue on OAT or switched to low-dose aspirin. The investigators intend to follow-up patients with thromboembolic event rate as primary and bleeding event rate as secondary end-points. The study is not expected to be completed until 2021. As we have discussed already, the use of aspirin as an alternative to OAT may not be a logical choice. Left atrial appendage occlusion is an alternative OAT-free substitute strategy aimed at reducing thromboembolic risk after AFCA and is in undergoing evaluation in a recently registered multi-centre randomised controlled trial against DOAC therapy.50

In counselling our patients as to the best approach for their long-term anticoagulation after AFCA the best we can do at present is be honest about the lack of robust data in this area and the dangers of inferring truth from case-cohort studies and observation. In doing so, we can be clear that ongoing AF and a high-risk score would strongly favour continuing OAT when possible. In the absence of recurrent AF particularly in patients who have developed risk factors some years after a successful AFCA it is probably reasonable to not re-institute OAT. For those patients between these two extremes we will have to continue to be honest and tell them that at the moment no-one knows what is correct and to do our best to help them make a decision that they are comfortable with. $\square$
1. Friberg $L$, Rosenqvist $M$, Lindgren $A$, et al. High prevalence of atrial fibrillation among patients with ischemic stroke Stroke. 2014;45:2599-605

2. Singer $D E$, Chang $Y$, Fang $M C$, et al. The net clinical benefit of warfarin anticoagulation in atrial fibrillation. Ann Intern Med. 2009;151:297-305

3. Pastori D, Pignatelli P, Menichelli D, et al. Integrated care management of patients with atrial fibrillation and risk of management of patients with atrial fibrillation and risk of
cardiovascular events: The ABC (Atrial fibrillation Better Care) cardiovascular events: The ABC (Atrial fibrillation Better Care)
Pathway in the ATHERO-AF Study Cohort. Mayo Clinic Proc. 2019;94:1261-7.

4. Kirchhof P, Benussi S, Kotecha D, et al. 2016 ESC Guidelines for the management of atrial fibrillation developed in collaboration with EACTS. Eur Heart J. 2016;37:2893-962.

5. Hart RG, Pearce LA, Aguilar MI. Meta-analysis: antithrombotic therapy to prevent stroke in patients who have nonvalvular atrial fibrillation. Ann Intern Med. 2007:146:857-67.

6. Connolly SJ, Ezekowitz MD, Yusuf S, et al. Dabigatran versus warfarin in patients with atrial fibrillation. N Engl I Med. 2009:361:1139-51.

7. Patel MR, Mahaffey KW, Garg Jet al. Rivaroxaban versus warfarin in nonvalvular atrial fibrillation. N Eng/ J Med. 2011:365:883-91. Granger CB, Alexander JH, MCMurray JJV, et al. Apixaban versus warfarin in patients with atrial fibrillation. N Eng/ J Med. versus warfarin in
2011:365:981-92.

2011;365:981-92.
Chai-Adisaksopha C, Crowther M, Isayama T, Lim W. The impact of bleeding complications in patients receiving target-specific oral anticoagulants: a systematic review and meta-analysis. Blood. 2014;124:2450-8.

10. Pisters R, Lane DA, Nieuwlaat R, et al. A novel user-friendly score (HAS-BLED) to assess 1-year risk of major bleeding in patients with atrial fibrillation: the Euro Heart Survey. Chest. patients with atrial

11. Gage BF. Waterman $A D$, Shannon W, et al. Validation of clinical classification schemes for predicting stroke: results from the National Registry of Atrial Fibrillation. JAMA. 2001;285:2864-70

12. Lip GYH, Nieuwlaat R, Pisters R, et al. Refining clinical risk stratification for predicting stroke and thromboembolism in atrial fibrillation using a novel risk factor-based approach: the Euro Heart Survey on atrial fibrillation. Chest. 2010;137:263-72.

13. Glotzer TV, Daoud EG, Wyse DG, et al. The relationship between daily atrial tachyarrhythmia burden from implantable device diagnostics and stroke risk: the TRENDS study. Circ Arrhythm Electrophysiol. 2009;2:474-80.

14. Al-Khatib SM, Thomas $L$, Wallentin $L$, et al. Outcomes of apixaban vs. warfarin by type and duration of atrial fibrillation:

5. Wyse DG, Waldo AL Di wyse DG, Waldo AL, DiMarco JP, et al. A comparison of rate control and rhythm control in patients with atrial fibrillation.

6. Mark DB, Anstrom KJ, Sheng S, et al. Effect of catheter ablation vs medical therapy on quality of life among patients with atrial fibrillation: The CABANA randomized clinical trial. JAMA. 2019;321:1275-85
17. Khan AR, Khan S, Sheikh MA, et al. Catheter ablation and antiarrhythmic drug therapy as first- or second-line therapy in the management of atrial fibrillation: systematic review and meta-analysis. Circ Arrhythm Electrophysiol. 2014;7:853-60.

18. Verma A, Champagne J, Sapp J, et al. Discerning the incidence of symptomatic and asymptomatic episodes of atrial fibrillation before and after catheter ablation (DISCERN AF): a prospective, multicenter study. JAMA Intern Med. 2013:173:149-56.

19. Calkins H, Hindricks G, Cappato R, et al. 2017 HRS/EHRA/ECAS/ APHRS/SOLAECE expert consensus statement on catheter and surgical ablation of atrial fibrillation: executive summary. J Inter Card Electrophysiol. 2017;50:1-55.

20. Badin A, Parr AR, Banga S, et al. Patients "and physicians" perceptions regarding the benefits of atrial fibrillation ablation. Pacing Clin Electrophysiol. 2017;40:362-71.

21. Lip GYH, Proclemer A, Dagres N, et al. Periprocedural anticoagulation therapy for devices and atrial fibrillation ablation. Europace. 2012;14:741-4.

22. Mardigyan V, Verma A, Birnie D, et al. Anticoagulation management pre- and post atrial fibrillation ablation: a survey of Canadian centres. Can J Cardiol. 2013;29:219-23.

23. Jarman JW, Hunter TD, Hussain W, et al. Stroke rates before and after ablation of atrial fibrillation and in propensity-matched controls in the UK. Pragmat Obs Res. 2017;8:107-18.

24. Bunch TJ, Crandall BG, Weiss JP, et al. Patients treated with catheter ablation for atrial fibrillation have long-term rates of death, stroke, and dementia similar to patients 
without atrial fibrillation. J Cardiovasc Electrophysiol. 2011;22:839-45

25. Hunter RJ, McCready J, Diab I, et al. Maintenance of sinus rhythm with an ablation strategy in patients with atrial fibrillation is associated with a lower risk of stroke and death. Heart. 2012;98:48-53.

26. Ghanbari H, Başer K, Jongnarangsin K, et al. Mortality and cerebrovascular events after radiofrequency catheter ablation of atrial fibrillation. Heart Rhythm. 2014;11:1503-11.

27. Joza J, Samuel M, Jackevicius CA, et al. Long-term risk of stroke and bleeding post-atrial fibrillation ablation. J Cardiovasc Electrophysiol. 2018;29:1355-62

28. Packer DL, Mark DB, Robb RA, et al. Effect of catheter ablation vs antiarrhythmic drug therapy on mortality, stroke, bleeding and cardiac arrest among patients with atrial fibrillation: The CABANA randomized clinical trial. JAMA. 2019:321:1261-74.

29. Saad EB, d'Avila A, Costa IP, et al. Very low risk of thromboembolic events in patients undergoing successful catheter ablation of atrial fibrillation with a CHADS2 score $\leq 3$ a long-term outcome study. Circ Arrhythm Electrophysiol. a long-term outcom

30. Themistoclakis S, Corrado A, Marchlinski FE, et al. The risk of thromboembolism and need for oral anticoagulation after successful atrial fibrillation ablation. J Am Coll Cardiol. 2010;55:735-43

31. Winkle RA, Mead RH, Engel G, et al. Discontinuing anticoagulation following successful atrial fibrillation ablation in patients with prior strokes. I Interv Card Electrophysiol. 2013;38:147-53

32. Atti V, Turagam MK, Viles-Gonzalez JF, Lakkireddy D. Anticoagulation after catheter ablation of atrial fibrillation: is it time to discontinue in select patient population? J Atr Fibrillation. 2018;11:2092.

33. Hart RG, Boop BS, Anderson DC. Oral anticoagulants and intracranial hemorrhage. Facts and hypotheses. Stroke.
1995;26:1471-7

34. Giugliano RP, Ruff CT, Braunwald E, et al. Edoxaban versus warfarin in patients with atrial fibrillation. N Eng/ J Med. 2013:369:2093-104

35. Chatteriee S, Sardar P Biondi-Zoccai G Kumbhani DJ. New oral anticoagulants and the risk of intracranial hemorrhage: traditional and Bayesian meta-analysis and mixed treatment comparison of randomized trials of new oral anticoagulants in atrial fibrillation. JAMA Neurol. 2013;70:1486-90.

36. Santarpia G, De Rosa S, Sabatino J, et al. Should we maintain anticoagulation after successful radiofrequency catheter ablation of atrial fibrillation? The need for a randomized study. Front Cardiovasc Med. 2017;4:85.

37. Zhang J-T, Chen K-P, Zhang S. Efficacy and safety of oral anticoagulants versus aspirin for patients with atrial fibrillation: a meta-analysis. Medicine (Baltimore). 2015:94:e409.

38. Healey IS, Connolly SI, Gold MR, et al. Subclinical atria fibrillation and the risk of stroke. N Eng/ J Med. 2012;366:120-9

39. Winkle RA, Mead RH, Engel G, et al. Atrial arrhythmia burden on long-term monitoring in asymptomatic patients late after atrial fibrillation ablation. Am J Cardiol. 2012;110:840-4.

40. Van Gelder IC, Healey JS, Crijns HJGM, et al. Duration of device-detected subclinical atrial fibrillation and occurrence of stroke in ASSERT. Eur Heart J. 2017;38:1339-44.

41. Osaka Y, Takigawa M, Takahashi A, et al. The proportion of symptomatic recurrence after catheter ablation of atria fibrillation in patients with a pacemaker for sick sinus syndrome. Indian Pacing Electrophysiol J. 2017;17:125-31.

42. Wechselberger S, Kronborg M, Huo Y, et al. Continuous monitoring after atrial fibrillation ablation: the LINQ AF study. Europace. 2018;20(FI_3):f312-20.

43. Wong JA, Conen D, Van Gelder IC, et al. Progression of device-detected subclinical atrial fibrillation and the risk of heart failure. J Am Coll Cardiol. 2018:71:2603-11.

44. Waks JW, Passman RS, Matos J, et al. Intermittent anticoagulation guided by continuous atrial fibrillation burden monitoring using dual-chamber pacemakers and implantable carlioverter-cefibrillators: Resuls from the Tailored Anticoaten for Non-Contits from the Tallored Anticoagulation for Non-Continuous Atrial Fibrillation (TACTIC-AF) pilot study. Heart Rhyth
2018:15:1601-7. 2018;15:1601-7.

45. Passman R, Leong-Sit P, Andrei A-C, et al. Targeted anticoagulation for atrial fibrillation guided by continuous rhythm assessment with an insertable cardiac monitor: The Rhythm Evaluation for Anticoagulation With Continuous Monitoring (REACT.COM) pilot study. J Cardiovasc Electrophysiol. 2016;27:264-70

46. Martin DT, Bersohn MM, Waldo AL, et al. Randomized trial of atrial arrhythmia monitoring to guide anticoagulation in patients with implanted defibrillator and cardiac resynchronization devices. Eur Heart J. 2015;36:1660-8.

47. Lopes RD, Alings M, Connolly SJ, et al. Rationale and design of the Apixaban for the Reduction of Thrombo-Embolism in Patients With Device-Detected Sub-Clinical Atrial Fibrillation (ARTESiA) trial. Am Heart J. 2017:189:137-45.

48. Kirchhof $\mathrm{P}$ Blank BF, Calvert $M$, et al. Probing oral anticoagulation in patients with atrial high rate episodes: Rationale and design of the Non-vitamin $\mathrm{K}$ antagonist Ora anticoagulants in patients with Atrial High rate episodes (NOAH-AFNET 6) trial. Am Heart J. 2017;190:12-8.

49. ClinicalTrials.gov. Identifier NCT02168829, Optimal Anticoagulation for Higher Risk Patients Post-Catheter Ablation for Atrial Fibrillation Trial (OCEAN) 2014. Available at: https://clinicaltrials.gov/ct2/show/NCT02168829 (accessed 18 September 2019).

50. ClinicalTrials.gov. Identifier NCT03795298, Comparison of Anticoagulation With Left Atrial Appendage Closure After AF Ablation (OPTION) 2019. Available at: http://clinicaltrials.gov/ct2/show/NCT03795298 (accessed 31 July 2019). 